\title{
Exploring Intramolecular Methyl-Methyl Coupling on a Metal Surface for Edge-Extended Graphene Nanoribbons
}

\author{
Zijie Qiüa (iD) \\ Qiang Sun ${ }^{\# b}$ (D) \\ Shiyong Wang ${ }^{\# b}$ (iD) \\ Gabriela Borin Barin ${ }^{b}$ (D) \\ Bastian Dumslaffa \\ Pascal Ruffieux ${ }^{b}$ (D) \\ Klaus Müllen*a,c (D) \\ Akimitsu Narita*a (i) \\ Roman Fasel ${ }^{* b, d}$ (D) \\ ${ }^{a}$ Max Planck Institute for Polymer Research, \\ Ackermannweg 10, 55128 Mainz, Germany \\ ${ }^{\mathrm{b}}$ Empa, Swiss Federal Laboratories for Materials Science \\ and Technology, nanotech@surfaces Laboratory, 8600 \\ Dübendorf, Switzerland \\ Department of Chemistry, University of Cologne, \\ Greinstr. 4-6, 50939 Cologne, Germany \\ ${ }^{d}$ Department of Chemistry and Biochemistry, University \\ of Bern, 3012 Bern, Switzerland \\ muellen@mpip-mainz.mpg.de; narita@mpip-mainz. \\ mpg.de; Roman.Fasel@empa.ch \\ \# These authors contributed equally to this work. \\ This paper is dedicated to Professor Peter Bäuerle on the \\ occasion of his $65^{\text {th }}$ birthday.
}
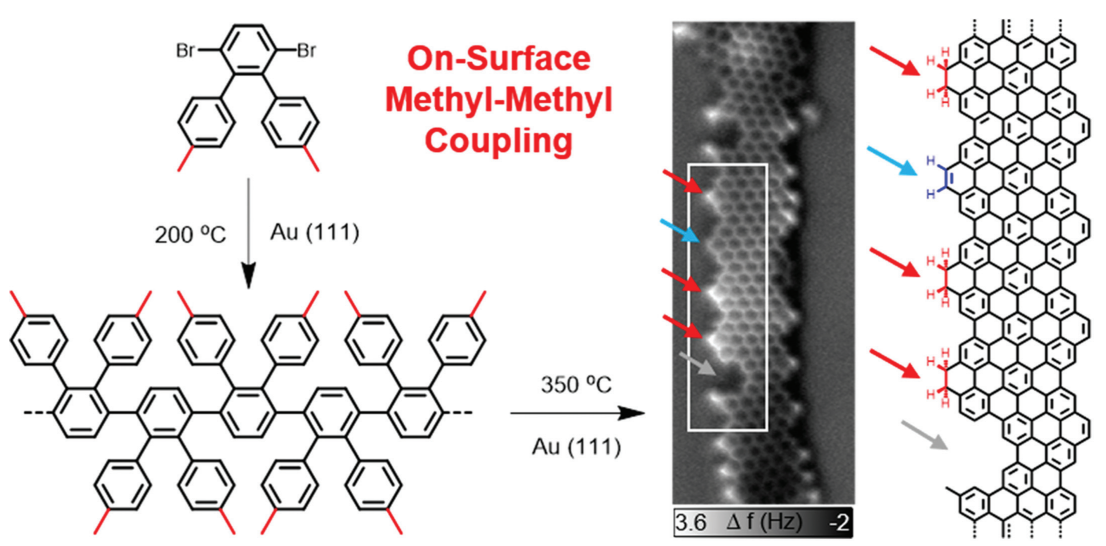

Received: 16.01.2021

Accepted after revision: 17.02.2021

DOI: 10.1055/s-0041-1726295; Art ID: om-21-0010sc License terms: (c)

(C) 2021. The Author(s). This is an open access article published by Thieme under the terms of the Creative Commons Attribution-NonDerivative-NonCommercial License, permitting copying and reproduction so long as the original work is given appropriate credit. Contents may not be used for commercial purposes, or adapted, remixed, transformed or built upon. (https://creativecommons.org/licenses/by-nc-nd/4.0/)

Abstract Intramolecular methyl-methyl coupling on Au (111) is explored as a new on-surface protocol for edge extension in graphene nanoribbons (GNRs). Characterized by high-resolution scanning tunneling microscopy, noncontact atomic force microscopy, and Raman spectroscopy, the methyl-methyl coupling is proven to indeed proceed at the armchair edges of the GNRs, forming six-membered rings with $\mathrm{sp}^{3}$ - or $\mathrm{sp}^{2}$-hybridized carbons.

Key words graphene nanoribbons, surface chemistry, edge extension, methyl-methyl coupling

\section{Introduction}

On-surface synthesis is an emerging approach to fabricate one-dimensional polymers and two-dimensional graphenic nanostructures with atomic precision. ${ }^{1-4}$ With the assistance of high-resolution surface-sensitive techniques and theoretical simulations, several classical organic reactions have been successfully realized via on-surface synthesis under ultrahigh vacuum (UHV) conditions. ${ }^{5-7}$ Among these are coupling protocols yielding products similar to Ullmann, ${ }^{8,9}$ Glaser, ${ }^{10,11}$ and Sonogashira reactions, ${ }^{12,13}$ as well as intramolecular processes like Bergman cyclization. ${ }^{14,15}$ Moreover, on-surface synthesis enables the construction of molecules that are challenging or not accessible via conventional solution chemistry. ${ }^{16-19}$ Therefore, the exploration of new on-surface chemistry is highly desirable to complement the limited tool kits and realize increasingly complex architectures.

Surface-assisted electrocyclic ring closure followed by loss of hydrogens is the final and critical step to construct fully conjugated carbon materials, such as nanographenes (NGs) and graphene nanoribbons(GNRs). ${ }^{20,21}$ Although the common aryl-aryl coupling alone does not allow the formation of zigzag edges, the simultaneous use of an on-surface methylaryl coupling enabled the formation of a zigzag GNR (6-ZGNR) in 2016. ${ }^{19}$ After polymerizing a U-shaped monomer $\mathbf{1}$ on a $\mathrm{Au}$ (111) surface, the obtained polymer was further annealed to $352^{\circ} \mathrm{C}$ to initiate the desired planarization process. Besides the cyclodehydrogenation between benzene rings, the methyl groups were also involved to achieve fully conjugated zigzag edges (Scheme 1A). Thereafter, the methyl-aryl oxidative ring closure has also been applied to produce an armchair GNR (7-AGNR) with extended edges (Scheme 1B). ${ }^{22-24}$ Besides hexagonal structures, methyl groups could be fused with the adjacent benzenes to also form five- ${ }^{25-28}$ or seven-membered rings ${ }^{29}$ through different precursor designs.

On the other hand, methyl-methyl coupling has been investigated on surfaces and furnished success in the 
(A)

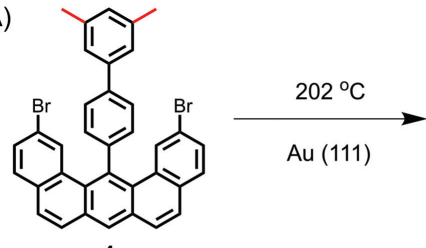

1

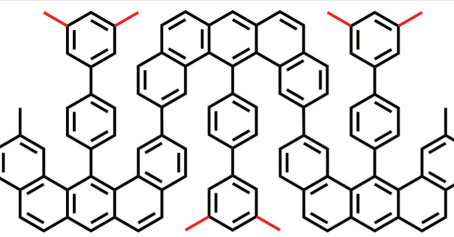

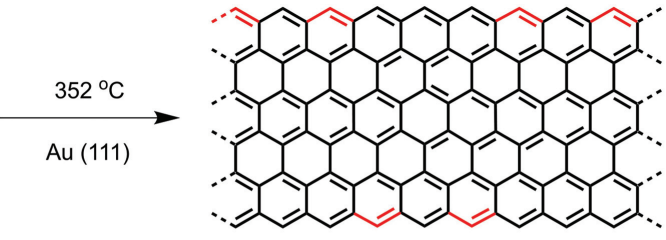

6-ZGNR

(B)
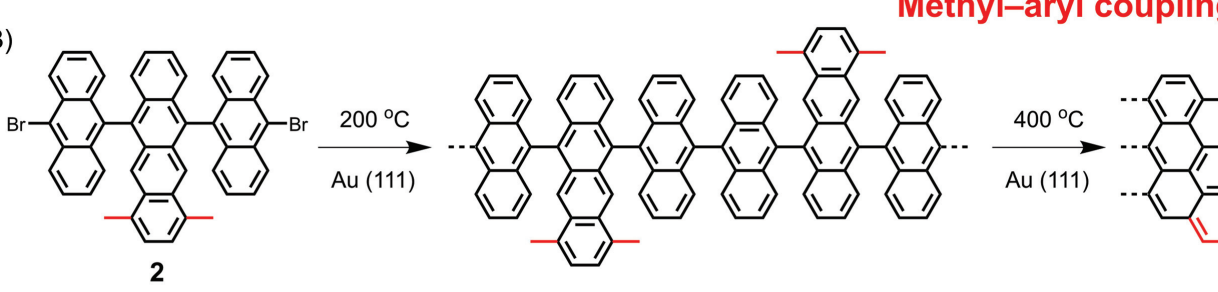

ing

(C) This work
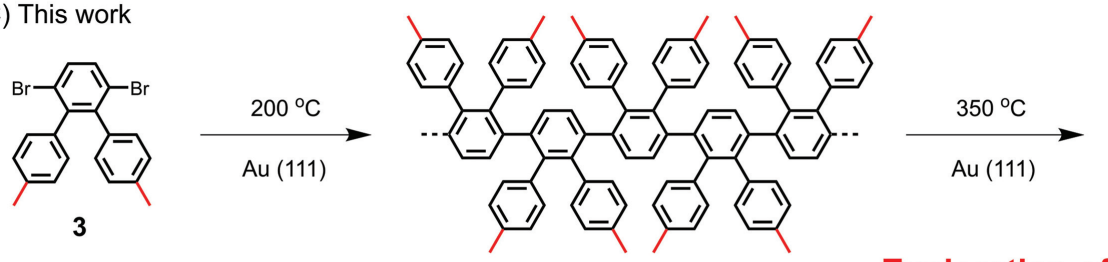

Exploration of methyl-methyl coupling

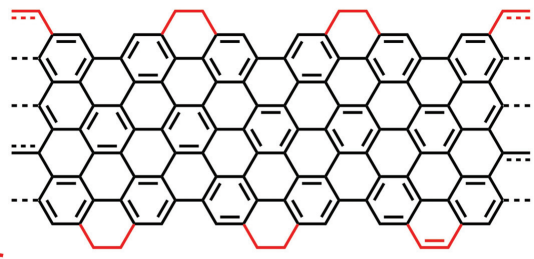

edge-extended 9-AGNR (9-eGNR)

Scheme 1 Synthetic routes to (A) 6-ZGNR and (B) edge-extended 7-AGNR via on-surface methyl-aryl couplings. (C) The on-surface synthesis involving methyl-methyl coupling in this work toward edge-extended 9-AGNR (9-eGNR) with both $\mathrm{sp}^{3}$ - and $\mathrm{sp}^{2}$-hybridized carbons on the edge. The methyl groups and the bonds formed through oxidative ring closure of methyl groups are highlighted in red.

intermolecular coupling between alkane chains or preactivated bromomethyl groups. $^{30-32}$ Recently, intramolecular methyl-methyl coupling has been achieved to construct circumcoronene, a hexagonal NG with six zigzag edges. ${ }^{33}$ However, the intramolecular coupling between two benzylic methyl groups has never been explored in GNRs, although it could potentially be developed as a powerful edge functionalization approach for structure engineering.

Therefore, in this work, we explored the on-surface methyl-methyl coupling using dimethyl substituted o-terphenyl $\mathbf{3}$ as the monomer towards the synthesis of edge-extended 9-AGNR (9-eGNR) (Scheme 1C). We expected that this approach would potentially provide fully conjugated 9-eGNR that is predicted to have electronic bands of topological origin. ${ }^{22}$ We found that the methyl-methyl coupling was indeed achieved along the ribbon at $350{ }^{\circ} \mathrm{C}$, furnishing the edge structures as characterized by high-resolution scanning tunneling microscopy (STM) and noncontact atomic force microscopy (ncAFM). However, not all of the ethanediyl bridges $\left(\mathrm{CH}_{2}-\mathrm{CH}_{2}\right)$ could undergo complete dehydrogenation towards conjugated alkenes even under further annealing at $440{ }^{\circ} \mathrm{C}$. The loss of aryl units was also observed, similar to the previously reported synthesis of pristine 9-AGNRs. ${ }^{34,35}$ These results shed light on the scope and limitation of intramolecular methyl-methyl coupling for future GNR synthesis.

\section{Results and Discussion}

The synthesis of the new monomer 3 was carried out as displayed in Scheme 2, adopting the procedure in previous reports. $^{36,37}$ Starting from commercially available 1,2-dibromobenzene (4), lithiation/silylation gave 1,4-disilyl intermediate $\mathbf{5}$ (Scheme 2). Subsequent Suzuki-Miyaura coupling of $\mathbf{5}$ with $p$-tolylboronic acid followed by bromination with $\mathrm{Br}_{2}$ at room temperature (RT) afforded monomer 3 . To guarantee the high purity required by the on-surface polymerization,

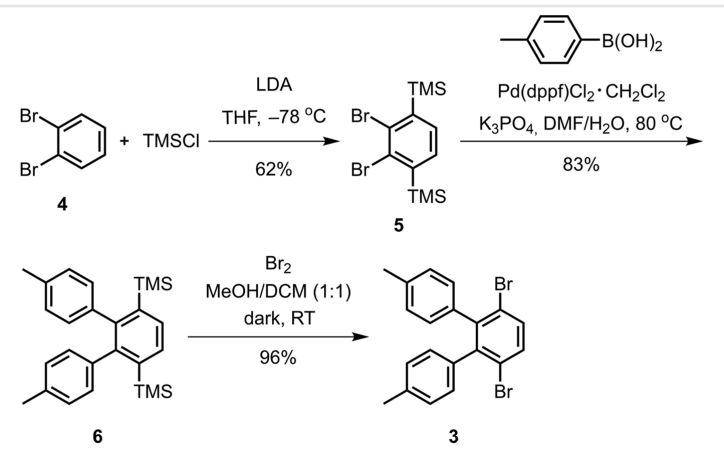

Scheme 2 Synthetic route to monomer 3. LDA = lithium diisopropylamide. $\mathrm{TMSCl}=$ trimethylsilyl chloride. $\mathrm{dppf}=1,1^{\prime}$ '-bis(diphenylphosphino)ferrocene. 
monomer $\mathbf{3}$ was recrystallized several times from methanol to completely remove the mono-brominated side-product, which could terminate the on-surface polymerization and limit the lengths of obtained GNRs. The contents of $\mathrm{C}$ and $\mathrm{H}$ atoms in elementary analysis were measured to be $57.8 \%$ and $3.8 \%$, respectively, which well matched with the calculated values for $\mathrm{C}_{20} \mathrm{H}_{16} \mathrm{Br}_{2}$ (C: $57.72 \%, \mathrm{H}$ : $3.88 \%$ ). Matrix-assisted laser desorption/ionization-time of flight (MALDI-TOF) mass spectrometry (MS) analysis of 3 was done using trans-2-[3-(4tert-butylphenyl)-2-methyl-2-propenylidene]malononitrile as the matrix and silver trifluoroacetate as the cationizing salt, thus leading to pseudo molecular ions where one silver cation was noncovalently attached to each molecule of 3 . An intense signal at $m / z=520.8669$ was observed with isotopic distribution patterns well-matched by the calculated spectrum (Figure S5, calculated value for $\mathrm{C}_{20} \mathrm{H}_{16} \mathrm{Br}_{2} \mathrm{Ag}^{+}$: 520.8664).

For investigating the on-surface synthesis, monomer 3 was first sublimed onto the Au (111) surface at RT under UHV conditions. Densely packed molecular islands of $\mathbf{3}$ were imaged by STM as displayed in Figure 1A. Due to the

(A)

(B)

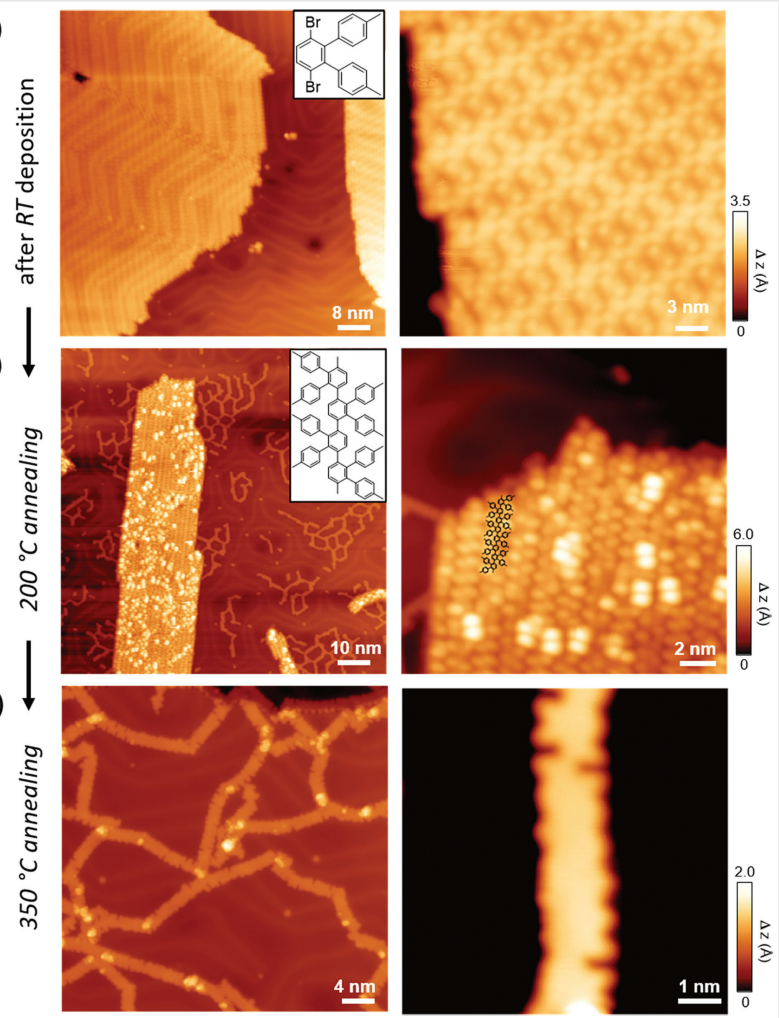

Figure 1 Large-scale (left) and zoom-in (right) STM images, as well as chemical structures of (A) monomer 3 sublimed on the Au (111) surface at RT, (B) the polyphenylene chain after annealing at $200^{\circ} \mathrm{C}$, and (C) the fully cyclodehydrogenated GNR after annealing at $350{ }^{\circ} \mathrm{C}$. Scanning parameters: $(A)$ left: $V_{\mathrm{s}}=-1 \mathrm{~V}, I_{\mathrm{t}}=100 \mathrm{pA}$; right: $V_{\mathrm{s}}=-1 \mathrm{~V}, I_{\mathrm{t}}=100$ pA; (B) left: $V_{\mathrm{s}}=-1 \mathrm{~V}, l_{\mathrm{t}}=10 \mathrm{pA}$; right: $V_{\mathrm{s}}=-1 \mathrm{~V}, I_{\mathrm{t}}=10 \mathrm{pA}$; (C) left: $V_{\mathrm{s}}=-0.5 \mathrm{~V}, I_{\mathrm{t}}=30 \mathrm{pA}$; right: $V_{\mathrm{s}}=-0.5 \mathrm{~V}, I_{\mathrm{t}}=30 \mathrm{pA}$. nonplanar geometry of $\mathbf{3}$, it is nontrivial to identify the individual molecules even from the high-resolution STM images. By gradually increasing the temperature to $200{ }^{\circ} \mathrm{C}$, as proposed in our earlier work, ${ }^{1}$ thermally activated debromination furnished biradical intermediates, which further polymerized to yield linear polyphenylene chains. At this stage, a different phase of the sample could be clearly observed in the STM image (Figure 1B). Because of the significant steric hindrance within the polymer, the central polyphenylene backbone tends to be flat and lie closer to the substrate than the branched methyl-phenyl groups. The brighter spots with the apparent height of $\sim 5$ A occasionally appearing in the STM image can be assigned to the tilting of some methyl groups out of plane, or the bromine atoms adsorbing on top of the molecule.

By further increasing the temperature to $350{ }^{\circ} \mathrm{C}$, the cyclodehydrogenation was triggered, fusing the benzene rings as well as inducing the coupling between the peripheral methyl groups (Figure 1C). A closer investigation using the bond-resolved nc-AFM imaging technique with a CO-functionalized $\operatorname{tip}^{38}$ was performed to reveal the fine structures at the atomic level. As clearly resolved in Figure 2C, three distinguishable edge structures, indicated by the coloured arrows, were formed after the reaction. The edge structures with bright features in the nc-AFM
(A)

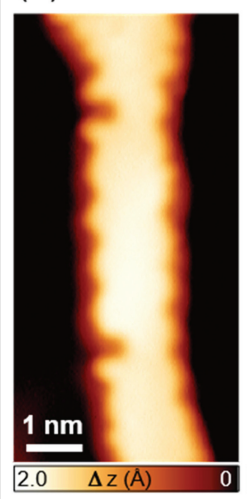

(B)

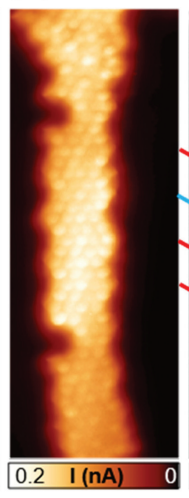

(C)

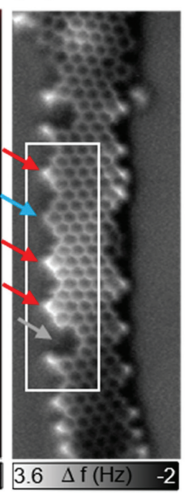

(D)

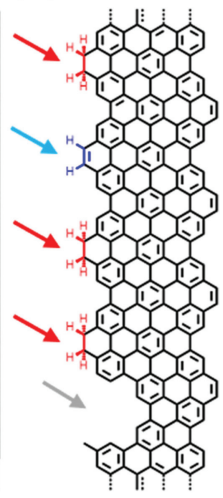

Figure 2 (A) High-resolution STM $\left(V_{\mathrm{s}}=0.01 \mathrm{~V}, I_{\mathrm{t}}=10 \mathrm{pA}\right)$ and (B) constant-height STM current image and the simultaneously acquired (C) nc-AFM image of the formed GNR $\left(V_{\mathrm{s}}=10 \mathrm{mV}\right)$. (D) Proposed chemical structure of the defective 9 -eGNR. Blue arrows: sp $^{2}$ hybridized carbon; red arrows: $\mathrm{sp}^{3}$ hybridized carbon; gray arrows: defects caused by the loss of aryl units.

image marked by the red arrows are attributed to the ethanediyl bridges with doubly hydrogenated $\mathrm{sp}^{3}$-hybridized carbons $\left(-\mathrm{CH}_{2}-\mathrm{CH}_{2}-\right)^{39}$ On the other hand, the formation of conjugated six-membered rings was also observed, leading to the $\pi$-extension of the armchair edge, as highlighted by the blue arrows. This result indicates 
that the methyl-methyl coupling can indeed be achieved on $\mathrm{Au}(111)$ surfaces under UHV conditions to afford the ethanediyl bridges, but the further dehydrogenation towards the fully conjugated structure is not efficient enough for the clean conversion. An approximate statistical analysis of the occurrence of the $-\mathrm{CH}=\mathrm{CH}$ - and $-\mathrm{CH}_{2}-\mathrm{CH}_{2}-$ motifs yields a ratio of 1:4 (another example of a typical 9-eGNR formed in our experiments is shown in Figure S6). Further annealing at $440{ }^{\circ} \mathrm{C}$ did not lead to the dehydrogenation of the ethanediyl bridges, suggesting a higher kinetic energy barrier and that different reaction mechanisms could be involved in the simultaneous formation of saturated and unsaturated C2 units.

Besides the coupling of methyl groups, the loss of aryl units was also observed during the cyclodehydrogenation process, which appeared as the "bite defects" (marked with gray arrows in Figure 2C). We note that these defects are not due to possible impurities in the precursor compound, but occur intrinsically during the on-surface cyclodehydration of polyphenylene. Similar defects were also observed during the previous synthesis of pristine 9-AGNRs using methylfree 0 -terphenyl-based monomers. ${ }^{34,35}$

Raman spectroscopy was applied for further characterization of the obtained 9-eGNR on $\mathrm{Au}(111)$. The radialbreathing-like mode (RBLM, $\sim 300 \mathrm{~cm}^{-1}$ ) displays very low intensity embedded in the background noise (Figure 3, marked by a dashed line), ${ }^{34}$ indicating that the width of the ribbons is not uniform. The $\mathrm{CH} / \mathrm{D}$ region of the Raman spectrum is a signature of the GNR's edge structure. The Raman spectrum of pristine 9-AGNRs exhibits two distinct narrow peaks at $1232 \mathrm{~cm}^{-1}(\mathrm{C}-\mathrm{H}$ bending mode) and $1332 \mathrm{~cm}^{-1}$ (D mode, peak width $\sim 15 \mathrm{~cm}^{-1}$ ), as displayed in Figure 3. ${ }^{40}$ However, a single broad peak at $1327 \mathrm{~cm}^{-1}$ was observed in the current 9-eGNR (peak width

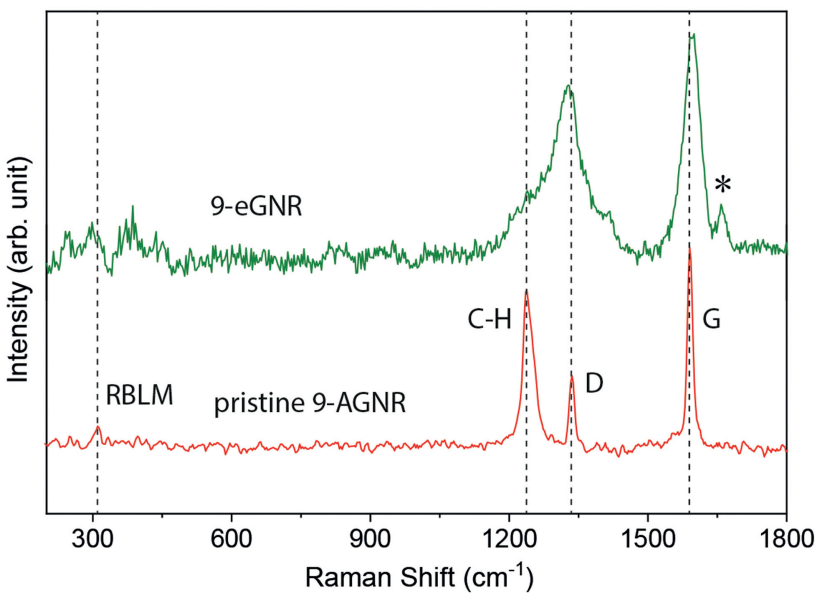

Figure 3 Raman spectra of the obtained 9-eGNR (green) and the pristine 9-AGNR (red) measured on Au(111) surfaces in air using $532 \mathrm{~nm}$ and $785 \mathrm{~nm}$ lasers, respectively. $\sim 100 \mathrm{~cm}^{-1}$ ), which is a clear indication of structural diversity. In addition, the appearance of a small peak around $1657 \mathrm{~cm}^{-1}$ (marked with *) seems similar to the D' mode associated with defects on GNR edges made by topdown approaches, as well as on graphenes and carbon nanotubes. ${ }^{41-43}$ More detailed investigations, for example by using tip-enhanced Raman spectroscopy methods, ${ }^{44}$ may thus provide further insights into the detailed chemical structures in the defective graphene materials.

\section{Conclusions}

In summary, methyl-methyl coupling was explored on a $\mathrm{Au}(111)$ surface under UHV conditions as a new synthetic approach for edge extension of AGNRs. As visualized by STM and nc-AFM, the coupling of methyl groups proceeded when heated to $350{ }^{\circ} \mathrm{C}$, mainly forming the ethanediyl bridges alongside fully conjugated six-membered rings. Considering the success of intramolecular methyl-methyl coupling in circumcoronene synthesis from dodecamethyl hexa-perihexabenzocoronene, ${ }^{33}$ we envision that the preplanarization might allow more efficient aromatization. Besides, a more reactive surface like $\mathrm{Cu}(111)$ is expected to achieve more $\mathrm{sp}^{2}$-hybridized carbons from the intramolecular methyl-methyl coupling. New monomer designs and further optimizations of dehydrogenation conditions will be conducted in the future to employ the methyl-methyl coupling for GNRs with complex architectures.

\section{Funding Information}

This work was supported by the Swiss National Science Foundation under Grant No. 200020_182015, the European Union's Horizon 2020 research and innovation programme under Grant Agreement No. 785219 (Graphene Flagship Core 2), the Max Planck Society, the Fund of Scientific Research Flanders (FWO) under EOS 30489208, the NCCR MARVEL funded by the Swiss National Science Foundation (51NF40-182892), and the Alexander von Humboldt Foundation.

\section{Supporting Information}

Supporting information for this article is available online at: https://doi.org/10.1055/s-0041-1726295.

\section{References And Notes}

(1) Cai, J.; Ruffieux, P.; Jaafar, R.; Bieri, M.; Braun, T.; Blankenburg, S.; Muoth, M.; Seitsonen, A. P.; Saleh, M.; Feng, X.; Müllen, K.; Fasel, R. Nature 2010, 466, 470. 
(2) Cai, J.; Pignedoli, C. A.; Talirz, L.; Ruffieux, P.; Söde, H.; Liang, L.; Meunier, V.; Berger, R.; Li, R.; Feng, X.; Müllen, K.; Fasel, R. Nat. Nanotechnol. 2014, 9, 896.

(3) Mishra, S.; Beyer, D.; Eimre, K.; Kezilebieke, S.; Berger, R.; Gröning, O.; Pignedoli, C. A.; Müllen, K.; Liljeroth, P.; Ruffieux, P.; Feng, X.; Fasel, R. Nat. Nanotechnol. 2020, 15, 22.

(4) Cirera, B.; Sánchez-Grande, A.; de la Torre, B.; Santos, J.; Edalatmanesh, S.; Rodríguez-Sánchez, E.; Lauwaet, K.; Mallada, B.; Zbořil, R.; Miranda, R.; Gröning, O.; Jelínek, P.; Martín, N.; Ecija, D. Nat. Nanotechnol. 2020, 15, 437.

(5) Shen, Q.; Gao, H.-Y.; Fuchs, H. Nano Today 2017, 13, 77.

(6) Clair, S.; de Oteyza, D. G. Chem. Rev. 2019, 119, 4717.

(7) Méndez, J.; López, M. F.; Martín-Gago, J. A. Chem. Soc. Rev. 2011, $40,4578$.

(8) Xi, M.; Bent, B. E. J. Am. Chem. Soc. 1993, 115, 7426.

(9) Lipton-Duffin, J. A.; Ivasenko, O.; Perepichka, D. F.; Rosei, F. Small 2009, 5, 592.

(10) Held, P. A.; Gao, H. Y.; Liu, L.; Mück-Lichtenfeld, C.; Timmer, A.; Mönig, H.; Barton, D.; Neugebauer, J.; Fuchs, H.; Studer, A. Angew. Chem. Int. Ed. Engl. 2016, 55, 9777.

(11) Gao, H. Y.; Wagner, H.; Zhong, D.; Franke, J. H.; Studer, A.; Fuchs, H. Angew. Chem. Int. Ed. Engl. 2013, 52, 4024.

(12) Kanuru, V. K.; Kyriakou, G.; Beaumont, S. K.; Papageorgiou, A. C.; Watson, D. J.; Lambert, R. M. J. Am. Chem. Soc. 2010, 132, 8081.

(13) Wang, T.; Huang, J.; Lv, H.; Fan, Q.; Feng, L.; Tao, Z.; Ju, H.; Wu, X.; Tait, S. L.; Zhu, J. J. Am. Chem. Soc. 2018, 140, 13421.

(14) Schuler, B.; Fatayer, S.; Mohn, F.; Moll, N.; Pavliček, N.; Meyer, G.; Peña, D.; Gross, L. Nat. Chem. 2016, 8, 220.

(15) Sun, Q.; Zhang, C.; Li, Z.; Kong, H.; Tan, Q.; Hu, A.; Xu, W. J. Am. Chem. Soc. 2013, 135, 8448.

(16) Zuzak, R.; Dorel, R.; Krawiec, M.; Such, B.; Kolmer, M.; Szymonski, M.; Echavarren, A. M.; Godlewski, S. ACS Nano 2017, 11, 9321.

(17) Krüger, J.; García, F.; Eisenhut, F.; Skidin, D.; Alonso, J. M.; Guitián, E.; Pérez, D.; Cuniberti, G.; Moresco, F.; Peña, D. Angew. Chem. Int. Ed. Engl. 2017, 56, 11945.

(18) Zuzak, R.; Dorel, R.; Kolmer, M.; Szymonski, M.; Godlewski, S.; Echavarren, A. M. Angew. Chem. Int. Ed. Engl. 2018, 57, 10500.

(19) Ruffieux, P.; Wang, S.; Yang, B.; Sánchez-Sánchez, C.; Liu, J.; Dienel, T.; Talirz, L.; Shinde, P.; Pignedoli, C. A.; Passerone, D.; Dumslaff, T.; Feng, X.; Müllen, K.; Fasel, R. Nature 2016, 531, 489.

(20) Qiu, Z.; Narita, A.; Müllen, K. Carbon nanostructures by macromolecular design-from branched polyphenylenes to nanographenes and graphene nanoribbons. Faraday Discuss. 2020. Doi: $10.1039 / \mathrm{d} 0 \mathrm{fd} 00023 \mathrm{j}$.

(21) Narita, A.; Wang, X. Y.; Feng, X.; Müllen, K. Chem. Soc. Rev. 2015, $44,6616$.

(22) Gröning, O.; Wang, S.; Yao, X.; Pignedoli, C. A.; Borin Barin, G.; Daniels, C.; Cupo, A.; Meunier, V.; Feng, X.; Narita, A.; Müllen, K.; Ruffieux, P.; Fasel, R. Nature 2018, 560, 209.

(23) Sun, Q.; Yao, X.; Gröning, O.; Eimre, K.; Pignedoli, C. A.; Müllen, K.; Narita, A.; Fasel, R.; Ruffieux, P. Nano Lett. 2020, 20, 6429.

(24) Rizzo, D. J.; Veber, G.; Jiang, J.; McCurdy, R.; Cao, T.; Bronner, C.; Chen, T.; Louie, S. G.; Fischer, F. R.; Crommie, M. F. Science 2020, 369, 1597.

(25) Di Giovannantonio, M.; Urgel, J. I.; Beser, U.; Yakutovich, A. V.; Wilhelm, J.; Pignedoli, C. A.; Ruffieux, P.; Narita, A.; Müllen, K.; Fasel, R. J. Am. Chem. Soc. 2018, 140, 3532.

(26) Di Giovannantonio, M.; Eimre, K.; Yakutovich, A. V.; Chen, Q.; Mishra, S.; Urgel, J. I.; Pignedoli, C. A.; Ruffieux, P.; Müllen, K.; Narita, A.; Fasel, R. J. Am. Chem. Soc. 2019, 141, 12346.
(27) Di Giovannantonio, M.; Chen, Q.; Urgel, J. I.; Ruffieux, P.; Pignedoli, C. A.; Müllen, K.; Narita, A.; Fasel, R. J. Am. Chem. Soc. 2020, 142, 12925.

(28) Jacobse, P. H.; McCurdy, R. D.; Jiang, J.; Rizzo, D. J.; Veber, G.; Butler, P.; Zuzak, R.; Louie, S. G.; Fischer, F. R.; Crommie, M. F. J. Am. Chem. Soc. 2020, 142, 13507.

(29) Lohr, T. G.; Urgel, J. I.; Eimre, K.; Liu, J.; Di Giovannantonio, M.; Mishra, S.; Berger, R.; Ruffieux, P.; Pignedoli, C. A.; Fasel, R.; Feng, X. J. Am. Chem. Soc. 2020, 142, 13565.

(30) Heinrich, B. W.; Ahmadi, G.; Müller, V. L.; Braun, L.; Pascual, J. I.; Franke, K. J. Nano Lett. 2013, 13, 4840.

(31) Zhong, D.; Franke, J.-H.; Podiyanachari, S. K.; Blömker, T.; Zhang, H.; Kehr, G.; Erker, G.; Fuchs, H.; Chi, L. Science 2011, 334, 213.

(32) Sun, Q.; Cai, L.; Ding, Y.; Ma, H.; Yuan, C.; Xu, W. Phys. Chem. Chem. Phys. 2016, 18, 2730.

(33) Telychko, M.; Li, G.; Mutombo, P.; Soler-Polo, D.; Peng, X.; Su, J.; Song, S.; Koh, M. J.; Edmonds, M.; Jelínek, P.; Wu, J.; Lu, J. Sci. Adv. 2021, 7, eabf0269.

(34) Talirz, L.; Söde, H.; Dumslaff, T.; Wang, S.; Sanchez-Valencia, J. R.; Liu, J.; Shinde, P.; Pignedoli, C. A.; Liang, L.; Meunier, V.; Plumb, N. C.; Shi, M.; Feng, X.; Narita, A.; Müllen, K.; Fasel, R.; Ruffieux, P. ACS Nano 2017, 11, 1380.

(35) Di Giovannantonio, M.; Deniz, O.; Urgel, J. I.; Widmer, R.; Dienel, T.; Stolz, S.; Sánchez-Sánchez, C.; Muntwiler, M.; Dumslaff, T.; Berger, R.; Narita, A.; Feng, X.; Müllen, K.; Ruffieux, P.; Fasel, R. ACS Nano 2018, 12, 74.

(36) Li, G.; Yoon, K. Y.; Zhong, X.; Zhu, X.; Dong, G. Chem. Eur. J. 2016 $22,9116$.

(37) Synthetic procedure for compound 3: Compound 6 (1.01 g, $2.5 \mathrm{mmol}$ ) was dissolved in a mixture of dichloromethane and methanol (300 mL, 1:1 ratio), and cooled to $0{ }^{\circ} \mathrm{C}$ under an argon atmosphere. In the absence of light, bromine $(0.39 \mathrm{~mL}$, $7.5 \mathrm{mmol}, 3$ equiv) was added dropwise so that the internal temperature did not increase rapidly. After stirring the mixture at room temperature overnight, the reaction was quenched with a saturated aqueous solution of sodium sulfite and extracted three times with dichloromethane. The combined organic phases were washed with brine and water and dried over magnesium sulfate. After the solvents were removed by rotary evaporation, the residue was purified by silica gel column chromatography using a mixture of hexane and dichloromethane (6:1) as the eluent. Monomer 3 was obtained as a colorless solid. Yield: $1.00 \mathrm{~g}$ (96\%). Recrystallization in ethanol was carried out several times before the on-surface study. Melting point: $169{ }^{\circ} \mathrm{C} .{ }^{1} \mathrm{H}$ NMR $(700 \mathrm{MHz}$, tetrachloroethane$\left.d_{2}\right): \delta 7.51(\mathrm{~s}, 2 \mathrm{H}), 6.95(\mathrm{~d}, J=7.7 \mathrm{~Hz}, 4 \mathrm{H}), 6.83(\mathrm{~d}, J=7.7 \mathrm{~Hz}, 4$ $\mathrm{H}), 2.24(\mathrm{~s}, 6 \mathrm{H}) .{ }^{13} \mathrm{C}$ NMR $\left(176 \mathrm{MHz}\right.$, tetrachloroethane- $\left.d_{2}\right): \delta$ 144.03, 137.06, 136.54, 132.62, 129.70, 128.25, 123.67, 21.42. MALDI-ToF-MS (positive): $m / z$ calculated for $\mathrm{C}_{20} \mathrm{H}_{16} \mathrm{Br}_{2} \mathrm{Ag}[\mathrm{M}]^{+}$: 520.8670, found 520.8669. Elemental analysis calculated for $\mathrm{C}_{20} \mathrm{H}_{16} \mathrm{Br}_{2}$ : C: 57.7, H: 3.9, found: C: 57.8, H: 3.9.

(38) Gross, L.; Mohn, F.; Moll, N.; Liljeroth, P.; Meyer, G. Science 2009, $325,1110$.

(39) Majzik, Z.; Pavliček, N.; Vilas-Varela, M.; Pérez, D.; Moll, N.; Guitián, E.; Meyer, G.; Peña, D.; Gross, L. Nat. Commun. 2018, 9, 1198.

(40) Borin Barin, G.; Fairbrother, A.; Rotach, L.; Bayle, M.; Paillet, M.; Liang, L.; Meunier, V.; Hauert, R.; Dumslaff, T.; Narita, A.; Müllen, K.; Sahabudeen, H.; Berger, R.; Feng, X.; Fasel, R.; Ruffieux, P. ACS Appl. Nano Mater. 2019, 2, 2184. 
(41) Dresselhaus, M. S.; Jorio, A.; Souza Filho, A. G.; Saito, R. Philos. Trans. R. Soc. London, Ser. A 2010, 368, 5355.

(42) Kampmann, F.; Scheuschner, N.; Terrés, B.; Jörger, D.; Stampfer, C.; Maultzsch, J. Ann. Phys. 2017, 529, 1700167.
(43) Eckmann, A.; Felten, A.; Verzhbitskiy, I.; Davey, R.; Casiraghi, C. Phys. Rev. B: Condens. Matter 2013, 88, 035426.

(44) Shiotari, A.; Kumagai, T.; Wolf, M. J. Phys. Chem. C 2014, 118, 11806. 